\title{
Design \& Validation Methodology applied to a roadster frame based on life prediction
}

\author{
Cristian Iorga, P.Eng., M.Sc. \\ Student \\ Cristian.Iorga@USherbrooke.ca
}

\author{
Alain Desrochers, P.Eng. Ph.D. \\ Professor \\ Alain.Desrochers@USherbrooke.ca
}

\author{
Department of Mechanical Engineering, \\ Faculty of Engineering, Université de Sherbrooke \\ 2500 Boul. de l'Université, J1K 2R1 \\ Sherbrooke (QC), Canada
}

\begin{abstract}
:
The recent increases in gasoline price have initiated a new thrust to reduce vehicle weight, hence creating a new market opportunity in the recreational product industry. At the same time, integration of both optimization and validation at the detailed design phase into the product development process has become key to achieving a product that meets the client needs from a price/performance/reliability perspective. Such integration also leads to more accurate requirements regarding the behavior of the structural components of a recreational vehicle. Therefore, to reach the objective of a weight reduction for the structural subsystem in a three wheels roadster project, a methodology that optimizes both frame geometry and material properties according to the following types of design criteria has been developed:

$>$ Structural criteria in order to support the specified loads;

$>$ Weight and cost criteria to assess some performance and market targets;

$>$ Qualitative criteria such as aesthetic, assembly or manufacturing.

One of the objectives of this paper is to outline a design and validation methodology that could be applied to the structural sub-systems of a recreational product with regard to all the design criteria established upstream in the product development process. This approach will converge into a creative, effective and profitable solution and will allow designers to offer a feedback on the client needs. The optimization process that the approach entail allows finding one or more combinations of parameters maximizing or minimizing a given design criterion, while the validation activities provide feedback to the designers in order to verify the calculations accuracy and the respect of all design criteria. According to the nature of the load cases identified, the proposed methodology has been applied to design and validate the frame of a three wheels roadster as part of a challenging multidisciplinary project involving students, professors and engineers from the recreational products industry.
\end{abstract}

